\title{
Asociación entre el estrés, el riesgo de depresión y el rendimiento académico en estudiantes de los primeros semestres de un programa colombiano de medicina
}

\author{
Hilda L. González-Olaya, Hernán D. Delgado-Rico, Mauricio Escobar-Sánchez, María Eugenia Cárdenas-Angelone
}

Introducción. Los estudiantes de medicina se enfrentan a múltiples factores que afectan su salud mental y rendimiento académico, sobre todo en los primeros semestres de la carrera.

Objetivo. Describir el comportamiento de la respuesta al estrés y la presencia de síntomas de depresión de importancia clínica a lo largo de un semestre académico en estudiantes del programa de medicina de la Universidad Autónoma de Bucaramanga y su asociación con el rendimiento académico.

Sujetos y métodos. Estudio prospectivo de cuatro cohortes de estudiantes, dos de primer semestre y dos de cuarto semestre, quienes fueron caracterizados sociodemográficamente y respondieron dos encuestas psicológicas (Zung y autopercepción de estrés), al inicio y al final de cada semestre. Se hizo un análisis descriptivo y se correlacionaron las variables de las encuestas, en ambos momentos del estudio, y con el rendimiento académico al final del curso, mediante $t$ de Student, análisis de regresión lineal y análisis estadístico bivariado y multivariado, utilizando el programa Stata 11.

Resultados. Se analizaron 216 estudiantes con una edad media de 18,4 años, procedentes en su mayoría del área metropolitana de Bucaramanga. No hubo diferencias entre los dos semestres y todas las variables psicológicas analizadas mediante los tests aplicados se correlacionaron con el rendimiento académico $(p \leq 0.05)$.

Conclusión. El presente estudio permite establecer que existe alta frecuencia de síntomas depresivos de importancia clínica y de autopercepción del estrés en los estudiantes de medicina, siendo ésta mayor en mujeres, así como una relación entre dichas variables psicológicas y el resultado académico.

Palabras clave. Depresión. Estrés. Estudiantes de medicina. Rendimiento académico.

Association among stress, depression risk and academic performance in students from the first semesters in a Colombian medical school

Introduction. Medical students have to deal with multiple factors that can affect their mental health and academic performance, especially in the first years of the medical program.

Aim. To describe the behavior of the stress response and the presence of depression symptoms of clinical importance in medical students at the Universidad Autónoma de Bucaramanga, throughout an academic semester; and their association with academic performance.

Subjects and methods. A prospective cohort study. Students from the first and fourth semesters were characterized sociodemographically and they answered two psychological tests (Zung self-rating depression scale and the Perceived Stress Scale) both, at the beginning and at the end of each semester. A descriptive analysis was performed, scales results at the two time points were correlated, between them, and also with academic performance, at the end of the academic semester. Student's $t$, linear regression analysis, univariate and multivariate analysis were used with Stata 11 software.

Results. 216 students participated in this study, with an average age of 18.4 years. Most of them came from Bucaramanga Metropolitan area. There were no statistical significant differences between the two semester's results, but there was a significant correlation between scales results and academic performance $(p \leq 0.05)$.

Conclusion. There is a high frequency of clinically significant depressive symptoms and self-perception of stress in medical students, being higher in women, and these results are related with students' academic performance.

Key words. Academic performance. Depression. Medical students. Stress.
Área de Ciencias Básicas Médicas; Facultad de Medicina (H.L. GonzálezOlaya, H.D. Delgado-Rico, M.E. Cárdenas-Angelone). Área de Salud Mental; Facultad de Ciencias de la Salud (M. Escobar-Sánchez). Programa de Medicina. Universidad Autónoma de Bucaramanga. Bucaramanga, Colombia.

Correspondencia:

Dra. Hilda Leonor González Olaya. Programa de Medicina. Universidad Autónoma de Bucaramanga. Calle 157, 19-55. Parque Campestre. Bucaramanga, Colombia.

E-mail:

hgonzalez4@unab.edu.co

Agradecimientos:

A la UNAB, por su apoyo en la

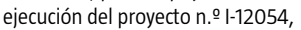
y al Dr. Víctor M. Herrera, por su colaboración en el análisis estadístico de los resultados.

Conflicto de intereses: No declarado.

Competing interests: None declared.

(C) 2014 FEM 


\section{Introducción}

Entre los problemas de salud mental, la tríada de estrés, ansiedad y depresión representa un reto para la salud pública tanto en Colombia como en el mundo. Según la Organización Mundial de la Salud, actualmente, cerca de 350 millones de personas sufren depresión y se cree que en el año 2020 esta afección se convertirá en la segunda causa global de discapacidad. En Colombia, según el Tercer Estudio Nacional de Salud Mental, la prevalencia de los episodios de depresión mayor es de 8,6\% para hombres y de 14,9\% para mujeres, es decir, en este país, como en el mundo, existe una mayor prevalencia de estrés y síntomas depresivos en mujeres que en hombres [1-3].

Se ha considerado que la prevalencia de los trastornos mentales es mayor en poblaciones con características particulares desde el punto de vista social, médico y ambiental. Entre las poblaciones analizadas, los estudiantes de medicina han sido un grupo de especial interés debido a las altas exigencias psicológicas, sociales y académicas que demanda esta carrera. Dentro de los factores académicos estresores se mencionan la excesiva carga de trabajo, las pruebas de evaluación académica, la incorporación de responsabilidades académicas nuevas y complejas, el autoritarismo docente y las pérdidas académicas, entre otros [4-6]. Sumados a los anteriores, se presentan factores de naturaleza no académica, como la dificultad para dar cumplimiento a las expectativas que el estudiante tiene respecto a la universidad y su carrera, la falta de apoyo económico, pérdidas afectivas, problemas de adaptación a la universidad, a otra ciudad y a otras familias, la separación de la familia de origen, menos horas de sueño y la asunción de otras responsabilidades personales [7].

La presencia de estrés y depresión entre los estudiantes de medicina se puede analizar desde dos perspectivas: la susceptibilidad propia antes de ingresar al programa de medicina, relacionada con aspectos biológicos y con la manera cómo los sujetos afrontan los problemas y las dificultades, y el estrés que se desarrolla a consecuencia de la exposición a los factores estresantes que acompañan el proceso de formación médica $[8,9]$. Adicionalmente, en Colombia, gran parte de los jóvenes universitarios inician esta etapa escolar aún en la adolescencia, y se ha descrito que la prevalencia de depresión con importancia clínica en adolescentes en el país es del 35-58\% [10,11].

Dentro de este contexto, la prevalencia de los síntomas depresivos y de estrés se ha documentado ampliamente en estudiantes de medicina. En el mundo, esta prevalencia es del 7-25\% para el trastorno depresivo y del 35-70\% para el estrés [12-14]. En Colombia, múltiples programas de medicina han realizado estudios para determinar la prevalencia de los trastornos de salud mental entre sus estudiantes y han hallado rangos amplios de prevalencia para la presencia de síntomas depresivos, del 25-56\% [15-19].

Los niveles inusualmente altos de estrés y depresión en estudiantes de medicina tienen implicaciones importantes que se extienden más allá de la educación médica, pues se ha observado que los profesionales de esta rama de la salud presentan tasas de suicidio más altas que la población general y otros profesionales, y cada vez son más frecuentes las publicaciones acerca del síndrome de agotamiento (burnout) no sólo entre estudiantes de medicina, sino entre los médicos [20-22].

Dentro de los instrumentos que con mayor frecuencia se utilizan para evaluar la presencia de síntomas depresivos están la escala de depresión de Beck, las entrevistas estructuradas y la escala de Zung. Esta última se emplea como un instrumento de tamización o diagnóstico en diferentes poblaciones con relativa validez y fiabilidad, que permite detectar síntomas de depresión con importancia clínica. En Colombia, y específicamente en Bucaramanga, Díaz et al [23] validaron la escala original (20 preguntas), propusieron una escala abreviada (10 preguntas) y encontraron para ambas una adecuada consistencia interna, sensibilidad y especificidad como instrumento de tamización en población de estudiantes universitarios.

Igualmente, la evaluación del estrés se puede realizar mediante diferentes escalas, y una de las más utilizadas es la escala de estrés percibido. Se diseñó con el propósito de conocer hasta qué punto las personas perciben como estresantes los eventos de la vida cotidiana, para uso en muestras poblacionales con al menos enseñanza secundaria [24]. Hay disponibles dos versiones de este instrumento: la original incluye 14 puntos (APE-14), y una versión más breve, 10 puntos (APE-10). Ambas muestran una excelente consistencia interna y estructura bidimensional en población de habla inglesa. CampoArias et al validaron esta escala en una muestra de mujeres universitarias de una universidad pública de Bogotá (Colombia), encontrando igualmente una excelente consistencia interna y aceptable estructura factorial para ambas versiones [25].

El efecto que tienen el estrés y la depresión sobre el rendimiento académico se ha documentado ampliamente en la literatura científica. Aunque dicho rendimiento es producto del proceso de evaluación llevado a cabo durante un período académico, en la mayor parte de las instituciones educativas se refle- 
ja básicamente en el indicador cuantitativo de la nota o en la categoría de aprobar o no el curso o el semestre. Sus determinantes y variables asociadas han sido objeto de gran interés, sobre todo porque un deficiente desempeño desemboca en mortalidad académica y deserción estudiantil, y por su asociación con la salud mental de los estudiantes [20-26].

La asociación entre estrés y trastornos afectivos con el rendimiento académico se ha analizado en ambas direcciones, es decir, los trastornos afectivos pueden conducir a un mal rendimiento académico, pero a su vez, un rendimiento académico inferior al esperado en una persona susceptible puede inducir síntomas depresivos, ansiedad y estrés. Los efectos negativos que produce el estrés y la depresión sobre el rendimiento académico pueden ser explicados por sus efectos sobre el desempeño cognitivo. Se ha demostrado que estos estados dificultan el rendimiento en tareas que requieren atención dividida, memoria de trabajo, adecuada y rápida recuperación de la información de la memoria, y toma de decisiones. Estos efectos parecen estar determinados por la evaluación individual de las demandas y de los recursos ante una situación, la relación entre el estresor y la tarea, y los estilos de afrontamiento, entre otros [27].

En el modelo tradicional de enseñanza de la medicina utilizado en la mayor parte de las escuelas de medicina colombiana, y caracterizado por un ciclo básico seguido del ciclo clínico, se ha observado que la mortalidad académica es mayor en el primero de estos dos [20]. Particularmente, en la Universidad Autónoma de Bucaramanga (UNAB), la mortalidad comunicada se ha caracterizado por ser más alta en los semestres primero y cuarto.

Con base en lo anterior, el objetivo del presente estudio fue evaluar el grado de estrés percibido y la prevalencia de síntomas depresivos de importancia clínica en estudiantes de medicina de la UNAB que cursan el primer y cuarto semestres, y determinar si el rendimiento académico se correlaciona con estos resultados.

\section{Sujetos y métodos}

Se realizó un estudio descriptivo y prospectivo de dos cohortes de estudiantes de primer semestre y dos cohortes de cuarto semestre del programa de medicina de la UNAB durante los dos semestres académicos del año 2012. Un total de 216 estudiantes se incluyeron en el estudio, cuando de manera voluntaria ellos, o bien sus representantes legales (si eran menores de edad), consentían de manera escrita su participación. Se realizaron las mismas determinaciones tanto al inicio (semana 1) como al final del semestre (semana 19) en curso, con el fin de establecer diferencias entre dos momentos clave del desarrollo académico del estudiante. Las variables a estudiar se obtuvieron a partir de un formato de información sociodemográfica y dos encuestas psicológicas que respondían los participantes en los dos momentos descritos. Las encuestas psicológicas fueron el test de Zung abreviado (de 10 preguntas, para medir síntomas depresivos de importancia clínica, cuyo punto de corte fue de 22) y el test APE, también su versión abreviada (de 10 preguntas, cuyo punto de corte para considerar como alto fue de 24). Estas dos encuestas han sido validadas previamente por investigadores nacionales en población universitaria colombiana y evalúan aspectos relevantes de carácter emocional, motivacional y social que se relacionan con la presencia de síntomas depresivos importantes y la autopercepción que se tiene del estrés en el momento en que se aplican. Al término de cada semestre se recopilaron las calificaciones finales del curso como variable de rendimiento académico y se estratificaron de acuerdo con el promedio general del curso.

Los datos fueron registrados y tabulados en una base para posteriormente analizarlos estadísticamente con el programa Stata 11 . Se realizó un análisis descriptivo de todas las variables del estudio con porcentajes y medidas de tendencia central y dispersión con sus respectivos intervalos de confianza del 95\% (IC 95\%). Las asociaciones entre las variables se establecieron mediante regresión lineal múltiple, calculando los coeficientes de correlación acorde con el tipo de variable. Para contrastar medias entre grupos de individuos en un punto de tiempo empleamos la prueba $t$ de Student y su extensión para muestras pareadas cuando el interés del análisis fue evaluar cambio en los individuos entre dos puntos de tiempo. Igualmente, para dichas comparaciones estimamos la media de las diferencias y su respectivo IC 95\%. Se empleó una regresión lineal múltiple para evaluar de manera simultánea la asociación entre diferentes predictores (demográficos y psicológicos) y la calificación final que fue el desenlace principal del estudio. En general, se construyeron dos tipos de modelos de regresión para predecir la calificación final: una, incluyendo los predictores medidos tanto en la primera como en la segunda evaluación, y otra, incluyendo el cambio del predictor entre evaluaciones, ajustado por el resultado del predictor medido en la línea de base. Finalmente, se retuvieron en el modelo de regresión final sólo aquellas variables cuyos coefi- 
Figura 1. Correlación entre los cambios observados según la media de las puntuaciones obtenidas en el test de Zung frente al test de autopercepción de estrés.

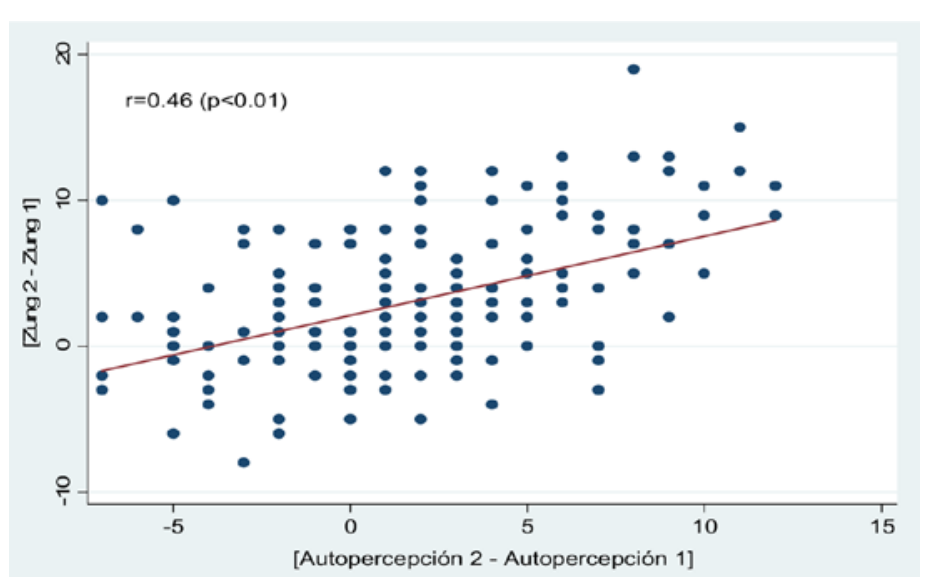

cientes (de regresión) fueron estadísticamente significativos al $5 \%$, es decir, con $p<0,05$. Se consideraron significativos los coeficientes de regresión con valores de $p<0,05$.

\section{Resultados}

En la tabla I se describen las características de los participantes. De los estudiantes que iniciaron el estudio, $9(8,8 \%)$ de primer semestre y $10(8,77 \%)$ de cuarto semestre no participaron en la segunda medición. En total se evaluaron los resultados de 415 encuestas, test de Zung y APE. En cuanto al sexo, se observó un mayor porcentaje de mujeres $(67,6 \%)$ y la media de edad de los participantes fue de 18,4 años. El 99\% de los participantes estaban solteros y en su mayoría procedían de Bucaramanga (95,83\%).

Los resultados del test de APE y del test de Zung se muestran en la tabla II. Los resultados permiten observar que, al final del semestre, el porcentaje de estudiantes con alta autopercepción de estrés y con síntomas depresivos de importancia clínica está alrededor del doble del porcentaje al inicio del mismo, y el promedio de los puntuaciones también aumentó, aunque en mayor medida para el test de Zung. De igual manera se observa que el mayor porcentaje de los participantes con alta autopercepción de estrés y síntomas depresivos correspondió al sexo femenino. Al correlacionar los resultados al inicio y al final en cada una de las dos escalas, se observa consistencia en el comportamiento. Es decir,
Figura 2. Distribución de calificaciones finales.

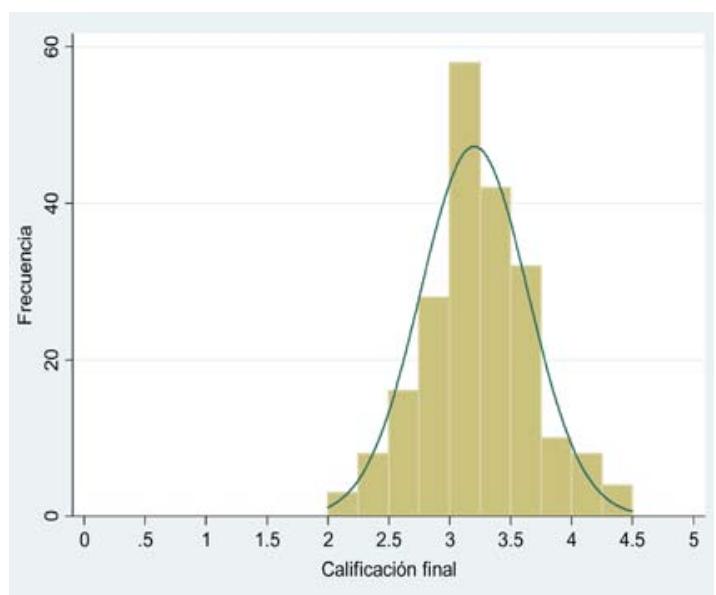

existió una tendencia al aumento en los valores promedio de los resultados, así como en el porcentaje de individuos que tuvieron un valor superior al corte. En otras palabras, la tendencia de la cohorte es a incrementar su autopercepción de estrés y síntomas depresivos a medida que avanza el semestre académico. Adicionalmente, se observa una relación directa entre las dos mediciones; por tanto, iniciar el curso con autopercepción de estrés y síntomas depresivos conlleva mayor probabilidad de terminarlo de la misma manera. Sin embargo, la magnitud de correlación es más fuerte para el test de Zung que para el test de APE.

De igual manera se establece una clara asociación entre los resultados observados en los dos tests: la mayor parte de los estudiantes que puntuaron alto en el test de APE también lo hicieron en el de Zung, y esto se evidencia en la figura 1, donde se muestra la gráfica de correlación entre los cambios observados en ambos tests (medición 2 - medición 1; $r=0,46 ; p<0,01)$.

Con relación a la calificación final obtenida en las dos asignaturas cursadas en primero y cuarto nivel del área de ciencias básicas médicas, se observa una distribución normal de las mismas (Fig. 2), con una nota mínima de 2,0, una nota máxima de 4,4 y una media de $3,2 \pm 0,4$. El $38 \%$ de los estudiantes tuvo calificación final entre 3,0 y 3,4. La nota $<3,0$ (que indica reprobación del curso) la obtuvo el 25\% de los estudiantes $(n=56), 27$ de primer nivel y 29 de cuarto. Al determinar si el rendimiento académico se correlaciona con los niveles de estrés per- 
Tabla I. Características sociodemográficas generales de los participantes $(n=216)$.

\begin{tabular}{lll}
\hline \multirow{2}{*}{ Participantes } & Primer semestre & $102(47,2 \%)$ \\
\cline { 2 - 3 } & Cuarto semestre & $114(52,7 \%)$ \\
\hline Sexo & Hombres & $70(32,4 \%)$ \\
\cline { 2 - 3 } & Mujeres & $146(67,6 \%)$ \\
\hline Edad (años) & Media \pm DE & $18,4 \pm 1,6$
\end{tabular}

cibido y la presencia de síntomas depresivos, los análisis estadísticos mostraron que un aumento en la diferencia de los valores (medición 2 - medición 1 ), tanto del test de Zung como en el test de APE, se correlaciona con una disminución en la nota final, pero la asociación es mayor con los resultados del test de Zung que con los del test de APE. Sin embargo, independientemente del cambio en la puntuación entre los dos momentos, se observa que comenzar con una puntuación elevada en el test de APE se relaciona con un mejor rendimiento académico.

En el análisis bivariado de la calificación definitiva (Tabla III) se observa que tanto la puntuación obtenida en la segunda encuesta del test de Zung y del test de APE, como la diferencia entre las dos puntuaciones de las dos pruebas psicológicas, permiten discriminar la probabilidad de reprobar (nota $<3,0$ ) o aprobar (nota $\geq 3,0$ ) el curso.

El modelo multivariado (Tabla IV) mostró que las puntuaciones en el test de Zung en la primera medición y su aumento en la segunda medición son las variables que en mayor medida inciden en el rendimiento académico, en tanto que un incremento de 10 puntos en el test de Zung inicial implica una disminución en la calificación final de aproximadamente 0,39 y un delta de 10 puntos implica una disminución aproximada de 0,47. En menor medida ocurre algo similar con la edad. Por su parte, la puntuación inicial del test de APE, así como su delta y pertenecer al sexo femenino, mostraron una influencia contraria sobre la calificación final, aumentándola levemente.

\section{Discusión}

El presente estudio evaluó estudiantes de medicina de dos semestres diferentes del área de ciencias básicas médicas del programa de medicina de la UNAB, primero y cuarto, en los cuales se abordan
Tabla II. Resultados del test de autopercepción del estrés (APE) y del test de Zung.

\begin{tabular}{|c|c|c|c|c|}
\hline & & Primera medición & Segunda medición & Diferencia (IC 95\%) \\
\hline \multirow{4}{*}{ APE } & $n$ & 216 & 199 & \\
\hline & Media $\pm \mathrm{DE}$ & $21,0 \pm 3,1$ & $22,7 \pm 3,9$ & $1,7(1,1-2,3)$ \\
\hline & Alto estrés percibido & $45(20,8 \%)$ & $81(41,0 \%)$ & \\
\hline & $\begin{array}{l}\text { Alto estrés } \\
\text { percibido (mujeres) }\end{array}$ & $31(68,8 \%)$ & $64(78,0 \%)$ & \\
\hline \multirow{4}{*}{ Zung } & $n$ & 216 & 199 & \\
\hline & Media $\pm \mathrm{DE}$ & $18,9 \pm 3,9$ & $21,9 \pm 4,9$ & $3,0(2,4-3,7)$ \\
\hline & $\begin{array}{l}\text { Presencia de síntomas } \\
\text { de importancia clínica }\end{array}$ & $49(23,79 \%)$ & $103(51,76 \%)$ & \\
\hline & Síntomas depresivos (mujeres) & $39(79,50 \%)$ & 75 (72,80\%) & \\
\hline
\end{tabular}

Tabla III. Asociación entre los tests psicológicos y aprobación/reprobación del curso.

\begin{tabular}{|c|c|c|c|c|}
\hline & & \multicolumn{2}{|c|}{ Calificación final } & \multirow{2}{*}{$p$} \\
\hline & & $\geq 3,0(n=157)$ & $<3,0(n=56)$ & \\
\hline & Primera medición & $18,8 \pm 3,8$ & $19,0 \pm 4,1$ & 0,714 \\
\hline \multirow[t]{3}{*}{ Zung } & Segunda medición & $20,9 \pm 4,6$ & $24,8 \pm 4,8$ & $<0,001$ \\
\hline & Diferencia & $2,1 \pm 4,3$ & $5,6 \pm 4,8$ & $<0,001$ \\
\hline & Primera medición & $21,1 \pm 3,0$ & $20,8 \pm 3,4$ & 0,612 \\
\hline \multirow[t]{2}{*}{ APE } & Segunda medición & $22,2 \pm 3,7$ & $24,1 \pm 4,2$ & 0,004 \\
\hline & Diferencia & $1,2 \pm 3,8$ & $3,0 \pm 4,4$ & 0,007 \\
\hline
\end{tabular}

respectivamente las asignaturas de 'Biología celular y molecular' y 'Procesos patológicos'. Estos semestres comparten la característica histórica de ser los de mayor mortalidad académica en la institución y son referidos de manera general y no formal por los estudiantes como altamente estresantes. Estas características probablemente se relacionan con el hecho de que los resultados de las diferentes variables observadas no mostraran diferencias estadísticamente significativas, razón por la cual los datos se analizaron de manera unificada. Estudios similares sí han mostrado diferencias en los resultados obtenidos de acuerdo al semestre académico cursado, pero en general las observaciones apuntan a diferencias entre los semestres básicos y clínicos, en- 
Tabla IV. Modelo multivariado para el cambio en la calificación final.

\begin{tabular}{lcc}
\hline & \multicolumn{2}{c}{ Calificación final } \\
\cline { 2 - 3 } & Diferencia media & IC $95 \%$ \\
\hline Edad (por 10 años) & $-0,56$ & $-0,89$ a $-0,23$ \\
\hline Femenino & 0,23 & 0,11 a 0,34 \\
\hline Test de Zung primera medición (por 10 puntos) & $-0,39$ & $-0,55$ a $-0,22$ \\
\hline Delta a Zung (por 10 puntos) & $-0,47$ & $-0,61$ a $-0,33$ \\
\hline Test de APE primera medición (por 10 puntos) & 0,22 & 0,01 a 0,43 \\
\hline Delta a APE (por 10 puntos) & 0,04 & $-0,13$ a 0,21 \\
\hline a Diferencia de puntuaciones de la segunda frente a la primera evaluación. $R^{2}$ del modelo $=0,30$.
\end{tabular}

contrándose mayores niveles de estrés y de depresión en los primeros [28,29].

Aunque hay un gran número de comunicaciones acerca de la alta prevalencia de estrés en estudiantes de medicina -incluso hasta valores del $80 \%$ [4, 30]-, los estudios que han utilizado el test de APE en su versión extensa ubican el mayor porcentaje de estudiantes dentro del rango de estrés leve. En el presente estudio, el alto estrés percibido pasó del $21 \%$ al inicio del semestre académico al $41 \%$ al final de éste, por lo que tales hallazgos pueden considerarse de importancia, sobre todo al final del semestre.

$\mathrm{Al}$ analizar la prevalencia de síntomas depresivos en este estudio, el valor encontrado en la medición al inicio del semestre (23,79\%) está cercano a los comunicados en la mayor parte de los estudios realizados en diferentes programas de medicina de Colombia, como por ejemplo en la Universidad de Antioquia $(27,8 \%)$, Universidad del Valle $(36,4 \%)$, Universidad Pontificia Bolivariana de Medellín (21,6\%), Universidad de Caldas $(24,6 \%)$ y el CES de Medellín (29\%), en tanto que el valor encontrado al final del semestre $(51,76 \%)$ es similar al obtenido en los programas de medicina de la Universidad Militar Nueva Granada $(53,2 \%)$ y Universidad Javeriana $(46,95 \%)$ [15-19]. Sin embargo, estas comparaciones no son muy precisas, debido a diferencias en los planes de estudio, en las poblaciones estudiadas, en las mediciones utilizadas y en el diseño del estudio realizado.

La consistencia en los resultados de las dos pruebas psicológicas empleadas reafirma que ambas apuntan a evaluar el estado de ánimo de los participantes y que existe una estrecha relación entre estas dos entidades. En el estudio llama la atención el cambio en los resultados de las mismas al final del semestre. Es un hecho que, desde el punto de vista académico, el último período se asocia con factores estresores no presentes al inicio, como la cercanía de los exámenes finales, la incertidumbre acerca de la aprobación del semestre, el cumplimiento de las expectativas planteadas, sumado a la carga de responsabilidades y el cansancio acumulado. Otro factor que puede influir es que la media de edad en el estudio fue de 18 años y casi el 30\% de los participantes tenían 16-17 años. La adolescencia es una etapa de gran vulnerabilidad, en la cual convergen aspectos biológicos, sociales y culturales particulares que afectan a la salud física y mental y que pueden dificultar el afrontamiento de los estresores académicos $[10,11]$.

El presente estudio confirma hallazgos previos acerca de la mayor prevalencia de estrés y depresión en mujeres, lo cual se ha descrito tanto en la población general como en estudiantes de medicina $[1,31]$.

Los resultados de esta investigación buscan establecer estrategias que apunten hacia la mejora del bienestar físico y mental de los estudiantes de medicina, para que ello se refleje en un mejor rendimiento académico; de ahí que uno de los resultados más interesantes del estudio fue la asociación que se encontró entre los resultados de las dos pruebas con la calificación final del curso. Altos niveles de estrés y la presencia de síntomas de depresión pueden afectar funciones cognitivas como la memoria de trabajo, la toma de decisiones, la concentración y la atención, alterando así el desempeño académico [27]. Un importante estresor son los exámenes académicos, los cuales representan una forma de amenaza para muchos estudiantes, y aunque predecible y de corta duración, su impacto desfavorable en ellos ha sido sustentado en la bibliografía acerca del estrés [28]. Al comparar la asociación de los resultados de las dos pruebas aplicadas en este estudio con la calificación final destacan los del test de Zung, los cuales mostraron una mayor significación estadística, es decir, se encontró que los estudiantes que comienzan con síntomas depresivos de importancia clínica y su deterioro (delta en las mediciones) a lo largo del semestre se asociaron con un desenlace académico negativo. Es importante destacar también que el análisis multivariado mostró una asociación positiva entre iniciar el semestre con alta autopercepción de estrés y la calificación final. Esto puede indicar que este tipo de estrés se podría catalogar como euestrés, y ayudaría a que los estudiantes movilicen sus recursos y esfuerzos cognitivos, motivacionales y actitudinales para sacar adelante el semestre académico [4]. Una ventaja 
del presente estudio es que al ser de cohorte, permitió hacer las evaluaciones en dos momentos diferentes del semestre, lo que favoreció el análisis no solo transversalmente en cada uno de los momentos académicos descritos, sino que adicionalmente permitió un análisis longitudinal a lo largo del curso. En un diseño similar, Stewart et al [32] analizaron en estudiantes de primer año de medicina los factores que predecían el estrés. Encontraron que aquellos que manejaban las situaciones estresantes con estrategias de evitación, presentaban mayores niveles de ansiedad y de depresión. En la segunda medición, ocho meses después, también encontraron que bajos niveles académicos previos, puntuaciones altas en escalas de depresión y de ansiedad al inicio y poco optimismo podían predecir un mayor nivel de estrés.

En conclusión, el presente estudio permite establecer que existe una alta frecuencia de síntomas depresivos de importancia clínica y de autopercepción del estrés en los estudiantes de medicina de la UNAB al finalizar el semestre académico (se duplican los datos en relación con el inicio del mismo), y ésta es mayor en mujeres, así como una relación entre dichas variables psicológicas y el resultado de la calificación final del curso. Sin embargo, dado que sólo se evaluaron dos niveles del programa, y aunque entre ellos no hubo diferencia, los resultados no son extrapolables a todos los estudiantes de medicina, por lo que sería recomendable extender este estudio a todas las cohortes de la carrera. Por otra parte, como las escalas psicológicas utilizadas son autoadministradas, puede haber un sesgo de notificación. Finalmente, para futuros estudios con estudiantes universitarios, sería recomendable contar con una escala de prevalencia de estrés académico, la cual en la actualidad no existe validada en nuestro país.

\section{Bibliografía}

1. World Health Organization. Sixty-fifth World Health Assembly 2012. URL: http://www.who.int/mediacentre/ events/2012/wha65/journal/en/index4.html. [02.07.2013]

2. The WHO World Mental Health Survey Consortium. Prevalence, severity, and unmet need for treatment of mental disorders in the World Health Organization World Mental Health Surveys. JAMA 2004; 291: 2581-90.

3. Posada-Villa JA, Aguilar S, Magaña C, Gómez LC. Prevalencia de los trastornos mentales y uso de servicios: resultados preliminares del estudio nacional de salud mental. Colombia 2003. Rev Colombiana de Psiquiatría 2004; 33: 241-62.

4. Román-Collazo C, Ortiz-Rodríguez F, Hernández-Rodríguez Y. El estrés académico en estudiantes latinoamericanos de la carrera de medicina. Revista Iberoamericana de Educación 2008; 46: 1-8.

5. Dyrbye LN, Thomas MR, Shanafelt TD. Medical student distress: causes, consequences, and proposed solutions. Mayo Clin Proc 2005; 80: 1613-22.

6. Benbassat J, Baumal R, Chan S, Nirel N. Sources of distress during medical training and clinical practice: suggestions for reducing their impact. Med Teach 2011; 33: 486-90.

7. Jacobs G. Non academic factors affecting the academic success of Grenadian students at St. George's University. St. George's: University Centre; 2002.

8. Enns MW, Cox BJ, Sareen J, Freeman P. Adaptive and maladaptive perfectionism in medical students: a longitudinal investigation. Med Educ 2001; 35: 1034-42.

9. Dahlin M, Joneborg N, Runeson B. Stress and depression among medical students: a cross-sectional study. Med Educ 2005; 39: 594-604.

10. Campo-Arias A. Trastorno distímico en adolescentes: conceptos actuales. Pediatría Rural y Extrahospitalaria 2005; 35: 417-25.

11. Campo-Arias A, Dallos-Bareño CM, González-Rueda SJ, Rodríguez-Rodríguez DC, Sánchez-Herrera ZM, Díaz-Martínez LA. Consumo de cigarrillo y síntomas depresivos en estudiantes de Bucaramanga, Colombia. Archivos Argentinos de Pediatría 2007; 105: 12-6.

12. Yiu V. Supporting the well-being of medical students. CMAJ 2005; 172: 889-90.

13. Firth-Cozens J. Medical student stress. Med Educ 2001; 35: 6-7.

14. Schwenk T, Davis L. Depression, stigma, and suicidal ideation in medical students. JAMA 2010; 304: 1181-9.

15. Campo-Cabal G, Gutiérrez JC. Psicopatología en estudiantes universitarios de la Facultad de Salud, Univalle. Revista Colombiana de Psiquiatría 2001; 30: 351-8.

16. Amézquita ME, González RE, Zuluaga D. Prevalencia de la depresión, ansiedad y comportamiento suicida en la población estudiantil de pregrado de la Universidad de Caldas, año 2000. Revista Colombiana de Psiquiatría 2003; 32: 341-56.

17. Guavita-Falla P, Sanabria-Ferrand P. Prevalencia de sintomatología depresiva en una población estudiantil de la Facultad de Medicina de la Universidad Militar Nueva Granada. Revista de la Facultad de Medicina de la UNAL 2006; 54: 76-87.

18. Bermúdez SB, Durán MM, Escobar C, Morales A, Monroy SA, Ramírez A, et al. Evaluación de la relación entre rendimiento académico y estrés en estudiantes de medicina. MedUNAB 2006; 9: 198-205.

19. Montoya LM, Gutiérrez JA, Toro BE, Briñón MA, Rosas E, Salazar LE. Depresión en estudiantes universitarios y su asociación con el estrés académico. Rev CES Med 2010; 24: 7-17.

20. Frank E, Biola $H$, Burnett C. Mortality rates and causes among US physicians. Am J Prevent Med 2000; 19: 155-9.

21. Dyrbye LN, Massie FS Jr, Eacker A, Harper W, Power D, Duming SJ, et al. Relationship between burnout and professional conduct and attitudes among U.S. medical students. JAMA 2010; 304: 1173-80.

22. Schernhammer E. Taking their own lives: the high rate of physician suicide. N Engl J Med 2005; 352: 2473-6.

23. Díaz LA, Campo A, Rueda GA, Barros JA. Propuesta de una versión abreviada de la escala de Zung para depresión. Colomb Med 2005; 36: 168-72.

24. Cohen S, Kamarck T, Mermelstein R. A global measure of perceived stress. J Health Soc Behav 1983; 24: 385-96.

25. Campo-Arias A, Bustos-Leiton G, Romero-Chaparro A. Consistencia interna y dimensionalidad de la escala de estrés percibido (EEP-10 y EEP-14) en una muestra de universitarias de Bogotá. Aquichán 2009; 9: 271-80.

26. Vélez-Van Meerbeke A, Roa-González C. Factores asociados al rendimiento académico en estudiantes de medicina. Educ Med 2005; 8: 24-32.

27. LeBlanc VR. The effects of acute stress on performance: implications for health professions education. Acad Med 2009; 84 (Suppl): 25-33.

28. Martín-Monzón IM. Estrés académico en estudiantes universitarios. Apuntes de Psicología 2007; 25: 87-99.

29. Hernández JP. Ansiedad y depresión en estudiantes de medicina de la Universidad Pontificia Bolivariana. Medicina UPB 1996; 25: $177-80$. 
30. Marty C, Matías G, Figueroa M, Larrain D, Cruz C. Prevalencia de estrés en estudiantes del área de la salud de la Universidad de los Andes y su relación con enfermedades infecciosas. Revista Chilena de Neuropsiquiatría 2005; 43: 25-32.

31. Backovic DV, Zivojinovic JI, Maksimovic J, Maksimovic M.
Gender differences in academic stress and burnout among medical students in final years of education. Psychiatr Danub 2012; 24: 175-81.

32. Stewart SM, Lam TH, Betson CL, Wong CM. A prospective analysis of stress and academic performance in the first two years of medical school. Med Educ 1999; 33: 243-50. 